\title{
Maternal vaccination against H1N1 influenza and offspring mortality: population based cohort study and sibling design
}

\author{
Jonas F Ludvigsson, ${ }^{1}, 2$ Peter Ström, ${ }^{1}$ Cecilia Lundholm, ${ }^{1}$ Sven Cnattingius, ${ }^{3}$ Anders Ekbom, ${ }^{3}$ \\ Åke Örtqvist,, 45 Nils Feltelius, ${ }^{6}$ Fredrik Granath, ${ }^{3}$ Olof Stephansson 3,7
}

'Department of Medical Epidemiology, Karolinska Institutet, Sweden

2Department of Paediatrics, Örebro University Hospital, Sweden

${ }^{3}$ Clinical Epidemiology Unit, Department of Medicine Solna, Karolinska Institutet, Sweden ${ }^{4} U$ nit of Infectious Diseases, Department of Medicine, Karolinska Institutet, Sweden

${ }^{5}$ Department of Communicable Disease Control and Prevention, Stockholm County Council, Sweden

${ }^{6}$ Medical Product Agency, Uppsala, Sweden

7Department of Women's and Children's Health, Karolinska Institutet and Hospital, Sweden Correspondence to:

J F Ludvigsson

jonasludvigsson@yahoo.com

Cite this as: BMJ 2015;351:h5585 doi: 10.1136/bmj.h5585

Accepted: 09 October 2015

\begin{abstract}
STUDY QUESTION

What is the mortality in offspring of mothers who had influenza $A(H 1 N 1) p d m 09$ vaccination during pregnancy?

METHODS

This was a prospective population based cohort study in seven healthcare regions in Sweden based on vaccinations taking place between 2 October 2009 and 26 November 2010. H1N1 vaccination data were linked with pregnancy and birth characteristics and offspring mortality data in 275500 births (of which 1203 were stillbirths) from 137886 mothers. Of these offspring, 41183 had been exposed to vaccination with Pandemrix, a monovalent AS03 adjuvanted H1N1 influenza vaccine, during fetal life. A primary comparison group consisted of pregnancies of women who were not vaccinated during the same calendar period. In a second comparison, non-exposed siblings of infants prenatally exposed to vaccination were used as controls. Cox regression was used to estimate hazard ratios for stillbirth, early neonatal mortality (days 0-6 after birth), and subsequent mortality (beginning on day 7) in vaccinated versus nonvaccinated women, adjusting for mother's age at delivery, body mass index, parity, smoking, country of birth, and disposable income and for sex of offspring.

STUDY ANSWER AND LIMITATIONS

The results of this study suggest that AS03 adjuvanted H1N1 vaccination during pregnancy does not affect the risk of stillbirth, early neonatal death, or later mortality in the offspring. During follow-up, 1172 stillbirths, 380 early neonatal deaths, and 706 deaths thereafter occurred. Compared with general population controls, this corresponded to adjusted
\end{abstract}

\section{WHAT IS ALREADY KNOWN ON THIS TOPIC}

Several studies have reported a neutral relation between maternal H1N1 vaccination during pregnancy and risk of adverse fetal outcome, but data on long term mortality in offspring are lacking

Lack of adjustment for residual confounding factors (genetic and environmental) shared within families is a potential source of bias in earlier studies

\section{WHAT THIS STUDY ADDS}

H1N1 vaccination during pregnancy does not seem to influence offspring mortality after the neonatal period

This study confirms a neutral association between $\mathrm{H} 1 \mathrm{~N} 1$ vaccination during pregnancy and adverse fetal outcome, including when familial factors are taken into account hazard ratios of 0.83 ( $95 \%$ confidence interval 0.65 to 1.04) for stillbirth, 0.71 ( 0.44 to 1.14) for early neonatal death, and 0.97 (0.69 to 1.36) for later death. When siblings were used as controls, adjusted hazard ratios were 0.88 ( 0.59 to 1.30 ) for stillbirth, 0.82 ( 0.46 to 1.49 ) for early neonatal death, and 0.78 ( 0.52 to 1.19 ) for later death. Limitations of the study include lack of data on miscarriage before gestational week 22, inability to ascertain which mothers had pandemic flu during pregnancy, and lack of data on factors influencing the decision to vaccinate during pregnancy.

\section{WHAT THIS STUDY ADDS}

H1N1 vaccination during pregnancy is not associated with adverse fetal outcome or offspring mortality, including when familial factors are taken into account.

FUNDING, COMPETING INTERESTS, DATA SHARING This project was supported by grants from the Swedish Research Council and the Swedish Council for Working Life and Social Research. NF was employed at the Swedish Medical Product Agency at the time of the study.

\section{Introduction}

The World Health Organization declared influenza $\mathrm{A}(\mathrm{H} 1 \mathrm{~N} 1) \mathrm{pdm} 09$ to be a pandemic influenza in mid2009. ${ }^{1-3}$ Several pandemic vaccines were produced during this time; Sweden opted for the AS03 adjuvanted monovalent vaccine, Pandemrix (GlaxoSmithKline). This vaccine was offered free of charge to all Swedish residents.

Given evidence that pregnant women were especially prone to severe influenza, ${ }^{34}$ vaccination was recommended at any stage of pregnancy. We and others have since examined pregnancy and fetal outcomes in mothers who were vaccinated against H1N1. ${ }^{4-24}$ Most research suggests that $\mathrm{H} 1 \mathrm{~N} 1$ vaccination has few adverse effects on pregnancy outcomes. Some studies have even found an inverse relation between H1N1 vaccination and adverse pregnancy outcomes, potentially owing to treatment selection bias. Fell et al noted in their recent systematic review that risk estimates for adverse pregnancy outcomes often move closer to the null after adjustment for potential confounders such as education, income levels, smoking, parity, and comorbidity. ${ }^{25}$ However, no study has considered confounding by familial (that is, genetic and early environmental) factors.

Despite the abundance of research on influenza A(H1N1)pdm09 vaccination and pregnancy outcomes, we are unaware of studies exploring mortality in offspring beyond the first week of life (sometimes 
also included with stillbirths in the joint outcome "perinatal death"17).

The primary objective of this population based cohort study was to explore mortality in the offspring of mothers who received influenza $\mathrm{A}(\mathrm{H} 1 \mathrm{~N} 1)$ pdm09 vaccination. A secondary objective was to examine stillbirth, early neonatal death, and offspring mortality after taking familial factors into account. To do this we used a sibling design. This design is useful if vaccinated women differ systematically from women not opting for vaccination (for instance, through being more health aware, which would decrease the risk of complications or of having more comorbidities that would increase the risk of complications).

\section{Methods}

We linked individual data on influenza A(H1N1)pdm09 vaccinations (Pandemrix ${ }^{26}$ ) administered in 2009-10 to pregnant women with information on pregnancy and birth characteristics from the Swedish Medical Birth Register. We added offspring mortality data from the Swedish Cause of Death Register. As described in our

\begin{tabular}{|c|c|c|}
\hline Characteristic & $\begin{array}{l}\text { Vaccinated } \\
(n=41183)\end{array}$ & $\begin{array}{l}\text { Not vaccinated } \\
(\mathrm{n}=234317)\end{array}$ \\
\hline \multicolumn{3}{|l|}{ Mother's age at birth, years: } \\
\hline$<19$ & $188(0.5)$ & $2895(1.2)$ \\
\hline $19-24$ & $4016(9.8)$ & $41750(17.8)$ \\
\hline $25-29$ & $10790(26.2)$ & $72335(30.9)$ \\
\hline $30-34$ & $15570(37.8)$ & $77173(32.9)$ \\
\hline$\geq 35$ & $10619(25.8)$ & 40164 (17.1) \\
\hline \multicolumn{3}{|l|}{ Mother's body mass index*: } \\
\hline$<18.5$ & $828(2.0)$ & $5969(2.5)$ \\
\hline $18.5-<25$ & $24293(59.0)$ & $133481(57.0)$ \\
\hline $25-<30$ & $8892(21.6)$ & $49003(20.9)$ \\
\hline$\geq 30$ & $4208(10.2)$ & $22325(9.5)$ \\
\hline Missing data & $2962(7.2)$ & $23539(10.0)$ \\
\hline Male sex & $21293(51.7)$ & $120641(51.5)$ \\
\hline \multicolumn{3}{|l|}{ Parity: } \\
\hline 0 & $17402(42.3)$ & $113402(48.4)$ \\
\hline 1 & $16411(39.8)$ & $83356(35.6)$ \\
\hline$\geq 2$ & $7370(17.9)$ & $37559(16.0)$ \\
\hline \multicolumn{3}{|l|}{ Smoking*: } \\
\hline Non-smoker & 37825 (91.8) & $205495(87.7)$ \\
\hline 1-9 cigarettes/day & $1711(4.2)$ & $13009(5.6)$ \\
\hline$\geq 10$ cigarettes/day & $486(1.2)$ & $4205(1.8)$ \\
\hline Missing data & $1161(2.8)$ & $11608(5.0)$ \\
\hline Mother born in Sweden & $33353(81.0)$ & $173268(73.9)$ \\
\hline Disposable income $>\$ 25000$ & $30787(74.8)$ & $178113(76.0)$ \\
\hline \multicolumn{3}{|l|}{ Status at end of follow-up: } \\
\hline Dead (pregnancy) & $115(0.3)$ & $1057(0.5)$ \\
\hline Dead (neonatal) $\dagger$ & $31(0.1)$ & $349(0.1)$ \\
\hline Dead (childhood) $\ddagger$ & $57(0.1)$ & $649(0.3)$ \\
\hline Alive & $40910(99.3)$ & 231668 (98.9) \\
\hline Emigrated & $70(0.2)$ & $594(0.3)$ \\
\hline Mean (SD) gestational age, weeks & $39.8(1.9)$ & $39.7(2.0)$ \\
\hline Mean (SD) age at end of follow-up, years & $4.1(0.3)$ & $6.4(4.0)$ \\
\hline
\end{tabular}

study on risk of neurological and immune related diseases in vaccinated people, ${ }^{27}$ we obtained vaccination data from seven Swedish regions (the counties of Kalmar, Östergötland, Stockholm, Värmland, and Norrbotten plus the healthcare regions of Västra Götaland and Skåne). These regions comprise around $61 \%$ of the Swedish population. ${ }^{27}$

In Sweden, Pandemrix vaccinations were given free of charge from October 2009 through 2010. High risk groups (such as pregnant women) were actively encouraged to have vaccination and were prioritised for the early batches of the vaccine. Recording of vaccination was considered complete in four of the seven regions involved in this study. ${ }^{27}$ In these regions, registration of vaccinations was required for cost reimbursements. In the remaining three counties (Kalmar, Värmland, and Norrbotten, representing about $13 \%$ of the study population), registration of vaccination was done through a web based system that required personal informed consent. Because of lack of consent (and thereby lack of the recorded personal identity number), classification of exposure (vaccination: yes/no) was not possible in $16-22 \%$ of vaccinated people in these counties. We classified these people as unexposed in the main analyses of the study but excluded them in a subsequent sensitivity analysis.

Through the county specific databases, we obtained data on personal identity number, ${ }^{28}$ as well as date of vaccination. Unlike children, some of whom received two vaccinations, pregnant women received no more than one vaccination. To safeguard the integrity of the study participants, personal identity numbers were replaced by unique serial identification numbers before data elaboration.

\section{Participants}

We originally retrieved data on 279999 births (includes siblings) between 24 April 1980 and 31 December 2012. We excluded 1232 without data in the Medical Birth Register, 3156 who did not fulfil inclusion criteria (dead or not in study counties before 30 September 2009, or inconsistent migration information), and 111 for other reasons. Thus, the final study sample included 275500 births (of which 1203 were stillbirths) to 137886 women. Table 1 shows characteristics of the participants.

\section{Follow-up}

In the main analysis, we restricted the study participants ( $\mathrm{n}=121979)$ to births after 30 September 2009. We followed these participants from 22 completed gestational weeks until death or censoring (emigration or end of follow-up, which was 22 May 2014), with separate analysis for stillbirth, early neonatal death, and later death (that is, deaths from seven completed days to 4.6 years). For the sibling analysis, we used the whole cohort (279999); however, because comparisons were done within the family, only discordant siblings with at least one event within the family $(n=3801,1130$, and 2190) contributed to the analyses of stillbirth, neonatal death, and death in the subsequent period. 


\section{H1N1 vaccination}

The study exposure was vaccination against influenza A(H1N1)pdm09 (Pandemrix) at any stage of pregnancy. In all, 93156 women who gave birth during the study period were vaccinated with Pandemrix, of whom 44\% were vaccinated during pregnancy ( $\mathrm{n}=41183$ offspring). Control pregnancies were those for which no record existed of influenza A(H1N1)pdm09 vaccination during pregnancy in a pregnant woman giving birth in any of the seven healthcare regions of this study. We also divided vaccinations according to trimester of pregnancy at the time of vaccination (1-13 weeks, 14-26 weeks, and 27 weeks until delivery).

\section{Covariates}

Data on covariates came primarily from the Medical Birth Register. ${ }^{29}$ This registry contains prenatal and neonatal data on more than $98 \%$ of all births in Sweden since 1973. ${ }^{29}$ During the first prenatal visit, commonly occurring at the end of the first trimester, pregnant women are interviewed and examined by a midwife. ${ }^{30}$ They are asked about current smoking habits ( $0,1-9$, and $\geq 10$ cigarettes per day) and about medical, obstetric, and gynaecological history. Self reported information about women's height is recorded, and women are weighed. We calculated body mass index (as weight in kilograms/height in meters squared) and categorised it into four groups $(<18.5,18.5-<25,25-<30$, and $\geq 30) .{ }^{31}$ Information about parity is collected at the time of delivery $(1,2$, and $\geq 3)$. A small proportion of women had no data on body mass index $(9.6 \%)$ or smoking (4.6\%). Through the government agency Statistics Sweden, we obtained information on mothers' country of birth (categorised as born in Sweden or born outside Sweden) and disposable annual income $(<\$ 25000$ or $\geq \$ 25000)$.

\section{Sibling comparisons}

Sibling studies take familial factors into account when comparing exposed and unexposed participants. From the Medical Birth Register, we identified all siblings of infants prenatally exposed to the mothers' vaccinations during pregnancy. We were thus able to compare mortality between children with the same mother according to vaccination exposure. By comparing offspring of the same mother discordant for vaccination exposure, we by design adjusted for genetic and environmental factors shared by the siblings.

\section{Outcome measure}

Overall death was the main outcome measure and was examined on the basis of time of occurrence. We specifically studied death during pregnancy (stillbirth from 28 completed gestational weeks until 2008, and from 22 weeks in 2009 and onwards), newborn deaths during the first six days life (early neonatal death), and death thereafter (from seven completed days of life to 4.6 years).

\section{Patient involvement}

No patients were involved in setting the research question or the outcome measures; nor were they involved in the design and implementation of the study. There are no plans to involve patients in the dissemination of results.

\section{Statistics}

All analyses were done in two ways. Firstly, we considered all pregnancies ending after 30 September 2009 (start of the vaccination period) as independent observations. Secondly, we used all pregnancies during which vaccination occurred, together with all other pregnancies of the same woman. We used this latter approach in the within family analyses by conditioning on the mother (stratified Cox).

We used Cox regression to estimate hazard ratios for stillbirth, early neonatal mortality (days 0-6), and subsequent mortality (beginning on day 7) in vaccinated compared with non-vaccinated women, with fetal/ infant age as the study timescale, adjusting for mother's age at birth, body mass index, parity, smoking, country of birth, disposable income, and sex of offspring. We found no evidence of non-proportional hazards. In addition, we specifically examined risks of stillbirth, early neonatal death, and offspring mortality according to trimester of vaccination.

For the analysis of stillbirth, the exposure (vaccine during pregnancy or during the trimesters) was time dependent. We began follow-up at 22 (or 28) gestational weeks. Thus, if the mother was vaccinated before the start of follow-up, we considered the fetus to be exposed from the start; otherwise we considered the participant to be unexposed until the date of vaccination. All data presented in the results section represent adjusted estimates.

We defined statistical significance as $95 \%$ confidence intervals for risk estimates not including 1.0. We used $\mathrm{R}$ statistical software (version 3.1.1) to analyse data.

\section{Results}

Vaccinated mothers tended to be slightly older than non-vaccinated mothers (table 1 ); $42.3 \%$ of vaccinated mothers and $48.4 \%$ of non-vaccinated mothers were nulliparous. Vaccinated mothers were more often born in Sweden than non-vaccinated mothers. The mean gestational ages at birth were 39.8 and 39.7 weeks in vaccinated and non-vaccinated mothers. For the offspring mortality analysis, children were followed until a mean age of 4.1 years in the vaccinated cohort and 6.4 years in the non-vaccinated cohort. Among the unexposed pregnancies in siblings $(n=39314), 31496$ (80.1\%) took place before the pregnancy exposed to Pandemrix, and 7818 (19.9\%) took place after the index pregnancy.

During pregnancy, 1172 stillbirths, 380 deaths in the early neonatal period, and 706 deaths after the early neonatal period occurred (table 1). Compared with general population controls, offspring of vaccinated mothers were not at increased risk of stillbirth (adjusted hazard ratio $0.83,95 \%$ confidence interval 0.65 to 1.04 ), early neonatal death $(0.71,0.44$ to 1.14$)$, or later death (0.97, 0.69 to 1.36$)$. When we used siblings as controls, corresponding hazard ratios were 0.88 (0.59 to 1.30), 0.82 (0.46 to 1.49 ), and 0.78 (0.52 to 1.19 ) (table 2 ). 


\begin{tabular}{|c|c|c|c|c|}
\hline & \multicolumn{2}{|l|}{ Population } & \multicolumn{2}{|l|}{ Sibling } \\
\hline & $\begin{array}{l}\text { Age adjusted hazard } \\
\text { ratio }(95 \% \mathrm{Cl})\end{array}$ & $\begin{array}{l}\text { Multivariable adjusted* } \\
\text { hazard ratio }(95 \% \mathrm{Cl})\end{array}$ & $\begin{array}{l}\text { Age adjusted hazard } \\
\text { ratio }(95 \% \mathrm{Cl})\end{array}$ & $\begin{array}{l}\text { Multivariable adjusted* } \\
\text { hazard ratio }(95 \% \mathrm{Cl})\end{array}$ \\
\hline \multicolumn{5}{|l|}{ Stillbirth } \\
\hline Overall & 0.83 (0.67 to 1.02) & 0.83 (0.65 to 1.04$)$ & $0.82(0.59$ to 1.15$)$ & $0.88(0.59$ to 1.30$)$ \\
\hline First trimester & 0.91 (0.68 to 1.23$)$ & 0.97 (0.72 to 1.33$)$ & $1.01(0.60$ to 1.70$)$ & 1.09 (0.61 to 1.97) \\
\hline Second trimester & 0.74 (0.54 to 1.03$)$ & 0.67 (0.47 to 0.97) & $0.81(0.46$ to 1.44$)$ & 0.77 (0.38 to 1.59) \\
\hline Third trimester & $0.82(0.53$ to 1.26$)$ & 0.82 (0.52 to 1.31$)$ & 0.59 (0.29 to 1.20$)$ & $0.68(0.31$ to 1.53$)$ \\
\hline \multicolumn{5}{|c|}{ Early neonatal death } \\
\hline Overall & 0.71 (0.47 to 1.06) & $0.71(0.44$ to 1.14$)$ & 0.65 (0.41 to 1.03) & 0.82 (0.46 to 1.49) \\
\hline First trimester & 1.04 (0.61 to 1.77$)$ & $0.98(0.52$ to 1.82$)$ & $0.82(0.41$ to 1.65$)$ & 1.07 (0.43 to 2.67) \\
\hline Second trimester & $0.57(0.29$ to 1.14$)$ & $0.64(0.31$ to 1.34$)$ & 0.42 (0.19 to 0.91) & 0.53 (0.20 to 1.37$)$ \\
\hline Third trimester & $0.47(0.21$ to 1.08$)$ & 0.48 (0.19 to 1.20$)$ & $1.00(0.35$ to 2.86$)$ & $1.24(0.32$ to 4.78$)$ \\
\hline \multicolumn{5}{|l|}{ Later death } \\
\hline Overall & 0.81 (0.59 to 1.11) & 0.97 (0.69 to 1.36) & 0.65 (0.46 to 0.93$)$ & 0.78 (0.52 to 1.19$)$ \\
\hline First trimester & $0.70(0.42$ to 1.16$)$ & $0.86(0.51$ to 1.47$)$ & $0.46(0.24$ to 0.87$)$ & $0.47(0.22$ to 1.01$)$ \\
\hline Second trimester & 0.91 (0.58 to 1.42) & 1.10 (0.69 to 1.76$)$ & 0.95 (0.54 to 1.68) & 1.44 (0.74 to 2.78$)$ \\
\hline Third trimester & 0.81 (0.49 to 1.35$)$ & $0.93(0.54$ to 1.60$)$ & $0.62(0.33$ to 1.19$)$ & 0.65 (0.30 to 1.39$)$ \\
\hline
\end{tabular}

None of the trimester specific risk estimates was statistically significant after adjustment for potential confounders, except for a decreased risk of stillbirth $(-33 \%)$ in women who were vaccinated in the second trimester. However, when we used siblings as controls, this decrease was no longer evident $(-23 \%$; $\mathrm{P}>0.05)$.

Excluding participants from the three regions Kalmar, Värmland, and Norrbotten did not influence our risk estimates more than marginally. Among the remaining 37584 pregnancies exposed to H1N1 vaccination, the hazard ratios for fetal death, early neonatal death, and later death were $0.80,0.76$, and 0.88 . When we used a sibling approach, the hazard ratios for these outcomes were similar to those in our main analysis $(0.80,0.88$, and 0.78$)$.

\section{Discussion}

This population based cohort study found no excess mortality in offspring of women who were vaccinated against influenza A(H1N1)pdm09 during pregnancy. Consistent with earlier data, we found no association between maternal vaccination and stillbirth or early neonatal mortality. ${ }^{25}$ Importantly, the associations between maternal vaccination and the risks of stillbirth, early neonatal mortality, and later mortality also remained close to null even when we used sibling controls. Our findings are reassuring in that a large proportion of Swedish women who were pregnant in 2009-10 were vaccinated against influenza A(H1N1)pdm09 despite limited knowledge about potential long term effects of Pandemrix. ${ }^{26}$ In all, our mortality study included more than 40000 children exposed to Pandemrix during fetal life.

\section{Comparison with other studies}

This study is one of the largest to date exploring the risk of stillbirth in women vaccinated against influenza A(H1N1)pdm09. Moreover, it is the first study to examine offspring mortality beyond the early neonatal period. A Swedish study with data suggesting that influenza $A(H 1 N 1) p d m 09$ vaccination was greatly under-ascertained (less than $12 \%$ of the mothers had a record of vaccination) used almost the same study population as this study when examining stillbirth. ${ }^{32}$ That study found a 23\% risk reduction for stillbirth among vaccinated women; we found a non-significant $17 \%$ risk reduction compared with the general population. Furthermore, several other large scale studies have reported an inverse relation between maternal vaccination and stillbirth (risk ratios $0.56,0.66,0.59$ $0.74,0.77$, and 0.79), ${ }^{79233233}$ although few of these inverse relations reached statistical significance. We suspected that the seemingly negative association might be due to selection bias and therefore examined stillbirth by using siblings as controls, which increased the hazard ratio somewhat (0.88). We conclude that maternal vaccination against influenza A(H1N1)pdm09 during pregnancy is unlikely to increase the risk of stillbirth. This conclusion is important, as recent data suggest that pregnant women with a clinical diagnosis of influenza are at increased risk of stillbirth. ${ }^{19}$

Although experts have proposed several mechanisms as to how vaccination might affect pregnancy outcomes (either decrease or increase the risk of adverse pregnancy outcome), we hypothesised that Pandemrix vaccination would not influence offspring mortality. Early life mortality is largely influenced by perinatal events, and considering that neither preterm birth nor stillbirth seems to follow influenza A(H1N1)pdm09vaccination, ${ }^{25}$ we thought it unlikely that offspring mortality would increase after H1N1 vaccination.

We studied offspring mortality according to time of follow-up (0-6 days after birth versus later). Mortality in the first days of life often differs from later mortality, as asphyxia has its predominant effect early in life. However, if H1N1 vaccination had any protective effect in offspring, it seems to have had its greatest effect shortly after birth. We believe that the trend towards a 
protective effect is a result of selection bias of mothers undergoing such vaccination, but a real effect cannot be ruled out considering that earlier data showed a protective effect against influenza in offspring of pregnant women undergoing vaccination..$^{3435}$ We found no significant association between vaccination in the third trimester and early neonatal death, either in the general population analysis (hazard ratio 0.48 ) or in the sibling analysis (hazard ratio 1.24). However, these estimates were based on few cases and results should be interpreted cautiously.

The neutral risk of offspring mortality beyond the perinatal period is the most novel finding in our study. As evidenced by the large number of papers on immune mediated diseases and influenza A(H1N1) pdm09 vaccination, people have feared that this vaccination might trigger an immune response. Such a response could take place not only in the mother but potentially also in the fetus. Our findings are comforting in that regard. We did not observe excess risk in either the general population control comparison (hazard ratio 0.97) or the sibling analysis (hazard ratio 0.78 ). The lack of adverse pregnancy outcomes is consistent with a recent questionnaire study by van der Maas that found no increase in infections recorded by general practitioners during the first year of life in infants exposed to H1N1 vaccination during pregnancy. ${ }^{36}$

\section{Strengths and limitations of study}

This study has several strengths. Firstly, we had access to detailed data on vaccination through regional vaccination registers, which precludes recall bias. Secondly, analyses were adjusted for potential confounders such as sex, parity, smoking, and body mass index. Thirdly, we believe that almost all pregnant women in the study area were included in our study. Fourthly, Swedish prenatal healthcare is almost exclusively funded by the public, ${ }^{37}$ and the influenza A(H1N1)pdm09 vaccination programme was free of charge, thereby counteracting selection bias. Furthermore, our study is one of the largest studies on influenza A(H1N1)pdm09 vaccination during pregnancy so far and included more than 300 offspring deaths during or after pregnancy. The large statistical power also allowed us to stratify for trimesters.

Among the weaknesses is our lack of data on miscarriage before gestational week 22, so we were unable to study whether this may have influenced the lack of association between H1N1 vaccination and stillbirth. Secondly, we were unable to ascertain which mothers had pandemic flu during pregnancy. Nor did we have data on factors influencing the decision to vaccinate during pregnancy, and we cannot rule out the possibility that residual confounding influenced our results. However, our use of sibling controls should control for at least some bias, and importantly the hazard ratio for stillbirth was neutral in that analysis.

\section{Conclusion}

Our results suggest that maternal H1N1 vaccination during any trimester of pregnancy has no adverse effect on mortality among offspring during pregnancy, in the early neonatal period, or in early childhood.

We thank Ingemar Persson for his previous work with the H1N1 cohort. Contributors: JFL conceived and designed the study, with input from the other authors. JFL wrote the first draft of the paper and supervised the project. JFL and SC obtained funding for the study. PS analysed the data, with support from CL and FG. All authors interpreted the data and contributed to the writing of the paper. All authors revised and approved the final version. JFL is the guarantor.

Funding: This project was supported by grants from the Swedish Research Council (Medicine, 2010-4202) and the Swedish Council for Working Life and Social Research (FAS: 2010-0923).

Competing interests: All authors have completed the ICMJE uniform disclosure form at www.icmje.org/coi_disclosure.pdf (available on request from the corresponding author) and declare: support for the submitted work as described above; NF was employed at the Swedish Medical Product Agency at the time of the study; no other relationships or activities that could appear to have influenced the submitted work.

Ethical approval: The study was approved by the Research Ethics Committee of Karolinska Institutet (2009/1952-31/4), which deemed that no individual informed consent was required.

Data sharing: Other researchers can apply for our data through the Swedish National Board of Health and Welfare.

Transparency: The lead author (the manuscript's guarantor) affirms that this manuscript is an honest, accurate, and transparent account of the study being reported; that no important aspects of the study have been omitted; and that any discrepancies from the study as planned (and, if relevant, registered) have been explained.

This is an Open Access article distributed in accordance with the Creative Commons Attribution Non Commercial (CC BY-NC 4.0) license, which permits others to distribute, remix, adapt, build upon this work non-commercially, and license their derivative works on different terms, provided the original work is properly cited and the use is non-commercial. See: http://creativecommons.org/licenses/ by-nc/4.0/.

1 Dawood FS, Jain S, Finelli L, et al. Emergence of a novel swine-origin influenza A (H1N1) virus in humans. N Engl J Med 2009:360:2605-15.

2 Garten RJ, Davis CT, Russell CA, et al. Antigenic and genetic characteristics of swine-origin 2009 A(H1N1) influenza viruses circulating in humans. Science 2009:325:197-201.

3 New influenza A (H1N1) virus: WHO guidance on public health measures, 11 June 2009. Wkly Epidemiol Rec 2009;84:261-4

4 Ludvigsson JF, Zugna D, Cnattingius S, et al. Influenza H1N1 vaccination and adverse pregnancy outcome. EurJ Epidemiol 2013;28:579-88

5 Moro PL, Broder K, Zheteyeva Y, et al. Adverse events following administration to pregnant women of influenza A (H1N1) 2009 monovalent vaccine reported to the Vaccine Adverse Event Reporting System. Am J Obstet Gynecol 2011;205:473.e1-9.

6 Huang WT, Chen WC, Teng HJ, et al. Adverse events following pandemic A (H1N1) 2009 monovalent vaccines in pregnant women-Taiwan, November 2009-August 2010. PLoS One 2011;6:e23049.

7 Fell DB, Sprague AE, Liu N, et al. H1N1 influenza vaccination during pregnancy and fetal and neonatal outcomes. Am J Public Health 2012;102:e33-40.

8 Pasternak B, Svanstrom H, Molgaard-Nielsen D, et al. Risk of adverse fetal outcomes following administration of a pandemic influenza A(H1N1) vaccine during pregnancy. JAMA 2012;308:165-74.

9 Pasternak B, Svanstrom H, Molgaard-Nielsen D, et al. Vaccination against pandemic A/H1N1 2009 influenza in pregnancy and risk of fetal death: cohort study in Denmark. BMJ 2012;344:e2794.

10 Dodds L, Macdonald N, Scott J, et al. The association between influenza vaccine in pregnancy and adverse neonatal outcomes. J Obstet Gynaecol Can 2012;34:714-20.

11 Heikkinen T, Young J, van Beek E, et al. Safety of MF59-adjuvanted A/ H1N1 influenza vaccine in pregnancy: a comparative cohort study. Am J Obstet Gynecol 2012;207:177.e1-8

12 Lin TH, Lin SY, Lin CH, et al. AdimFlu-S((R)) influenza A (H1N1) vaccine during pregnancy: the Taiwanese Pharmacovigilance Survey. Vaccine 2012;30:2671-5.

13 Tavares F, Nazareth I, Monegal JS, et al. Pregnancy and safety outcomes in women vaccinated with an ASO3-adjuvanted split virion H1N1 (2009) pandemic influenza vaccine during pregnancy: a prospective cohort study. Vaccine 2011;29:6358-65.

14 Omon E, Damase-Michel C, Hurault-Delarue C, et al. Non-adjuvanted 2009 influenza A (H1 N1)v vaccine in pregnant women: the results of a French prospective descriptive study. Vaccine 2011:29:9649-54. 
15 Mackenzie IS, MacDonald TM, Shakir S, et al. Influenza H1N1 (swine flu) vaccination: a safety surveillance feasibility study using self-reporting of serious adverse events and pregnancy outcomes. BrJ Clin Pharmacol 2012;73:801-1.

16 Oppermann M, Fritzsche J, Weber-Schoendorfer C, et al. A(H1N1) v2009: a controlled observational prospective cohort study on vaccine safety in pregnancy. Vaccine 2012;30:4445-52

17 Rubinstein F, Micone P, Bonotti A, et al. Influenza A/H1N1 MF59 adjuvanted vaccine in pregnant women and adverse perinatal outcomes: multicentre study. BM/ 2013;346:f393.

18 Richards JL, Hansen C, Bredfeldt C, et al. Neonatal outcomes after antenatal influenza immunization during the 2009 H1N1 influenza pandemic: impact on preterm birth, birth weight, and small for gestational age birth. Clin Infect Dis 2013;56:1216-22.

19 Haberg SE, Trogstad L, Gunnes N, et al. Risk of fetal death after pandemic influenza virus infection or vaccination. N Engl/ Med 2013;368:333-40.

20 Huang WT, Tang FW, Yang SE, et al. Safety of inactivated monovalent pandemic (H1N1) 2009 vaccination during pregnancy: a populationbased study in Taiwan Vaccine 2014:32:6463-8.

21 Trotta F, Da Cas R, Spila Alegiani S, et al. Evaluation of safety of A/ H1N1 pandemic vaccination during pregnancy: cohort study. BMJ 2014:348:g3361.

22 Luteijn JM, Brown MJ, Dolk H. Influenza and congenital anomalies: a systematic review and meta-analysis. Hum Reprod 2014;29:809-23.

23 Beau AB, Hurault-Delarue C, Vidal S, et al. Pandemic A/H1N1 influenza vaccination during pregnancy: a comparative study using the EFEMERIS database. Vaccine 2014;32:1254-8.

24 Chambers CD, Johnson D, Xu R, et al. Risks and safety of pandemic H1N1 influenza vaccine in pregnancy: birth defects, spontaneous abortion, preterm delivery, and small for gestational age infants. Vaccine 2013;31:5026-32.

25 Fell D, Platt R, Lanes A, et al. Fetal death and preterm birth associated with maternal influenza vaccination: systematic review. BJOG 2015;122:17-26
26 Medical Products Agency. [Benefits and risks of vaccinations with Pandemrix during pregnancy.] 2009. www.lakemedelsverket.se/ Alla-nyheter/NYHETER-2009/Nyttariskvardering-av-vaccination-medPandemrix-av-barn-och-gravida/.

27 Persson I, Granath F, Askling J, et al. Risks of neurological and immune-related diseases, including narcolepsy, after vaccination with Pandemrix: a population- and registry-based cohort study with over 2 years of follow-up. J Intern Med 2014;275:172-90.

28 Ludvigsson JF, Otterblad-Olausson P, Pettersson BU, et al. The Swedish personal identity number: possibilities and pitfalls in healthcare and medical research. Eur J Epidemiol 2009;24:659-67.

29 Cnattingius S, Ericson A, Gunnarskog J, et al. A quality study of a medical birth registry. Scand / Soc Med 1990;18:143-8

30 Cnattingius S, Villamor E, Johansson S, et al. Maternal obesity and risk of preterm delivery. JAMA 2013;309:2362-70.

31 World Health Organization. BMI classification. 2015. apps.who.int bmi/index.jsp?introPage=intro 3.html.

32 Kallen B, Olausson P. Vaccination against H1N1 influenza with Pandemrix ((R)) during pregnancy and delivery outcome: a Swedish register study. BJOG 2012:119:1583-90.

33 Sammon CJ, Snowball J, McGrogan A, et al. Evaluating the hazard of foetal death following $\mathrm{H} 1 \mathrm{~N} 1$ influenza vaccination; a population based cohort study in the UK GPRD. PLoS One 2012;7:e51734.

34 Zaman K, Roy E, Arifeen SE, et al. Effectiveness of maternal influenza immunization in mothers and infants. N Engl J Med 2008;359:1555-64

35 Madhi SA, Cutland CL, Kuwanda L, et al. Influenza vaccination of pregnant women and protection of their infants. N Engl J Med 2014;371:918-31.

36 Van der Maas N, Dijs-Elsinga J, Kemmeren J, et al. Safety of vaccination against influenza $\mathrm{A}(\mathrm{H} 1 \mathrm{~N} 1)$ during pregnancy in the Netherlands: results on pregnancy outcomes and infant's health: cross-sectional linkage study. BJOG 2015; published online 6 Mar.

37 Anell A. The public-private pendulum-patient choice and equity in Sweden. N Engl / Med 2015;372:1-4. 